Original

\title{
Tumor renal con trombo en la vena cava inferior. Manejo quirúrgico y pronóstico
}

\author{
Fernandes Denardi, Leonardo Oliveira Reis, Ricardo Reges M. Oliveira, Fábio Ferreira, \\ Ubirajara Ferreira
}

Sección de Urología Oncológica. Servicio de Urología. Universidad de Campinas. Unicamp-Campinas, Brasil

\begin{abstract}
Resumen
Objetivos: el objetivo del presente estudio fue evaluar las estrategias usadas para el manejo quirúrgico del cáncer de renal con extensión de trombo a la vena cava inferior y evaluar el pronóstico del paciente.

Métodos: Quince pacientes con cáncer renal y trombo en la vena cava inferior fueron sometidos a una nefrectomía radical y trombectomía y fueron evaluados de forma retrospectiva. Los pacientes fueron seguidos desde 0 a 36 meses (media de 9,5 meses). La tomografía computada con reconstrucción tridimensional fue usada para definir el nivel del trombo.

Resultados: La edad media fue de 54 años, con un rango de entre 16 y 79 años. El trombo fue clasificado como de nivel I $(13,5 \%)$, nivel II $(40 \%)$, nivel III $(26,5 \%)$ y nivel IV $(20 \%)$. La cirugía fue llevada a cabo mediante by pass cardiopulmonar en seis casos, tres de los cuales sin esternotomía. Hubo una muerte intraoperatoria (13\%) en un paciente que presentaba enfermedad metastásica con un trombo que alcanzaba la aurícula derecha. Los pacientes con ganglios positivos o enfermedad metastásica como presentación inicial tenían peor supervivencia global en la curva de Kaplan-Meier. Ambos, estadiaje tumoral $(\mathrm{p}=0,380)$, y extensión del trombo $(\mathrm{p}=0,174)$ no tuvieron relación con la supervivencia.

Conclusiones: A pesar de su morbilidad y mortalidad, la nefrectomía radical y la trombectomía deberían ser llevadas a cabo en casos de carcinoma renal con trombo tumoral, porque puede ofrecer la posibilidad de control local de la enfermedad. Los pacientes con ganglios linfáticos y enfermedad metastásica tienen peor pronóstico.
\end{abstract}

Palabras clave: Trombo vena cava inferior. Tumor renal. Pronóstico y manejo quirúrgico.

\section{Renal tumor with inferior vena cava thrombus. Surgical approach and prognosis \\ Abstract}

Objectives: The aim of this study was to evaluate strategies used for surgical management of renal cell carcinoma with tumoral thrombus extension in the inferior vena cava (IVC) and evaluate patient's prognosis.

Metods: Fifteen patients with renal tumor and IVC thrombus, underwent radical nephrectomy and thrombectomy, and were retrospectively evaluated. Patients were followed for 0 to 36 months (mean of 9.5 months). Computed tomography (CT) scan with three-dimensional reconstruction was used to define IVC thrombus level.

Results: The mean age was 54 years, range from 16 to 79 . The thrombus was classified as level I - 13.5\%, level II - 40\%, level III $-26.5 \%$ and level IV - 20\%. Surgery was performed with cardiopulmonary bypass (CPB) in six (40\%) cases, three $(50 \%)$ without sternotomy. There was one (13\%) death during the intraoperative period; this patient presented extensive metastatic tumor, with the thrombus reaching the right atrium. Patients with lymph node or metastatic disease at initial presentation had poorer overall survival in Kaplan-Meier curve. Both, tumor stage ( $\mathrm{p}=0.380$ ), and thrombus extension ( $\mathrm{p}$ $=0.174$ ) were not related to survival.

Conclusions: Despite its morbidity and mortality, radical nephrectomy with thrombectomy should be performed in case of renal cell carcinoma with tumoral thrombus, because it can offers the possibility of local control. Patients with lymph node and distant metastatic disease have poor prognoses.

Keywords: Inferior vena cava thrombus. Renal tumor. Prognosis, surgical approach.

L os tumores renales suponen entre el 2 y $3 \%$ de todas las enfermedades malignas diagnosticados. En 2002, 18.700 nuevos casos fueron diagnosticados en Estados Unidos con 7500 muertes debidos al carcinoma renal de células claras ${ }^{1}$. Entre el 4 y $15 \%$ de estos tumores tienen trombo tumoral asociado en la vena renal o en la vena cava inferior (VCI) y el tipo histológico más frecuente en los tumo- res con trombo tumoral asociado es el Carcinoma de Células Renales (CCR) ${ }^{5-9}$.

Aproximadamente un tercio de los pacientes ya se presentan con enfermedad metastásica a distancia en el momento del diagnóstico y ello conlleva peor pronóstico ${ }^{10-12}$.

La presencia de trombo tumoral está asociada a peor comportamiento biológico pero no necesaria- 
mente a peor pronóstico ${ }^{1-2}$. La extensión tumoral parece tener mayor efecto pronóstico cuando se comparada con la presencia de trombo tumoral en el sistema venoso ${ }^{11-14}$.

Entre el 40 y $75 \%$ de los pacientes con trombo tumoral pueden ser curados mediante cirugía. Los tumores confinados al riñón, libres de trombo en los vasos tienen mejor pronóstico. De otra manera, los tumores que invaden la grada perirrenal, con ganglios linfáticos o con invasión directa de las paredes de los vasos tienen peor pronóstico ${ }^{13,14}$.

La cirugía radical es el único tratamiento curativo potencial en los casos de tumor renal con trombo, porque no hay otra terapia alternativa eficaz. Algunos autores publican la mejoría supervivencia global en pacientes sometidos a resección radical del tumor renal así como del trombo comparado con pacientes tratados únicamente con nefrectomía ${ }^{5-8}$.

El estudio preoperatorio radiológico para determinar el tumor y la extensión del trombo es esencial para definir el abordaje quirúrgico. La extensión craneal del trombo es esencial para definir la necesidad de by pass cardiopulmonar (BCP).

La tomografía computada es el estándar para definir el diagnóstico y ocasionalmente la Resonancia Magnética Nuclear es importante para detallar mejor el trombo tumoral y la evaluación de la invasión de la pared vascular ${ }^{9}$.

El objetivo del presente estudio fue evaluar estrategias usadas para el manejo quirúrgico del cáncer renal con extensión tumoral a la vena cava inferior (VCI). El resultado peri operatorio, mortalidad y supervivencia global de acuerdo a la técnica quirúrgica fueron comparadas.

\section{MÉTODOS}

\section{Pacientes}

Quince pacientes con carcinoma de células renales y trombo tumoral en la vena cava inferior a quienes se practicó nefrectomía radical y trombectomía fueron estudiados de forma retrospectiva entre abril de 1999 y marzo de 2006. Pacientes con metástasis viscerales o linfáticas no fueron excluidos. En todos los casos la cirugía fue realizada por el mismo cirujano y todos los pacientes firmaron el consentimiento informado.

La información demográfica de los pacientes, síntomas y estadio clínico fueron evaluados preoperatoriamente. Los tumores fueron clasificados de acuerdo a la clasificación TNM de 2002.

\section{Evaluación preoperatorio}

En todos los casos, la evaluación preoperatorio incluyó tomografía computada de abdomen y tórax (TC) con reconstrucción tridimensional y Doppler de vena cava para definir la extensión del tumor y la extensión craneal del trombo. Los pacientes que presentaban circunstancias más complejas (invasión de la aurícula) fueron evaluados con Resonancia Magnética. El límite craneal del trombo fue clasificado de acuerdo al sistema de Neves y Zincke y de acuerdo a este sistema, el nivel I era renal (el trombo tenía una extensión de menos de $5 \mathrm{~cm}$. por encima del ostium de la vena renal) el nivel II era infrahepático (debajo de la vena cava infrahepática), nivel III retrohepático (el trombo invadía la porción intrahepática de la vena cava inferior, pero por debajo del diafragma) y nivel IV era auricular (encima del diafragma, pudiendo alcanzar la aurícula derecha).

\section{Técnica quirúrgica}

La exposición se logró mediante una incisión de chevron bilateral y cuando fue necesario se extendió al apex xifoides para una mejor exposición de órganos (incisión de Mercedes), la cual garantiza una gran exposición. Tras ello los órganos y la cavidad fueron examinados.

Inicialmente, el pedículo renal fue abordado, la arteria renal ligada y seccionada dejando la vena renal intacta. Tras ello, la vena renal fue disecada verificando la extensión del trombo tumoral.

Para trombos de nivel I no fueron necesarias maniobras adicionales. El clamp de Satinsky se colocó obliterando parcialmente la vena cava inferior, bombeando el trombo hacia el riñón y la vena cava inferior con un borde de seguridad. La vena cava inferior fue suturada con sutura de polipropileno de 3.0. En cuanto al trombo de nivel II, la vena cava infrarrenal así como la vena cava infradiafragmática, el pedículo hepático y la vena renal opuesta fueron clampadas con clamps vasculares.

Esto permitió el control de la circulación colateral dejando las venas lumbares sin control. Un retractor automático fue necesario para una adecuada exposición y permite una participación más activa del equipo quirúrgico. Después de controlar la circulación colateral se realizó una pequeña cavotomía anterior para la extracción del trombo. Tras ello, se seccionó la vena renal y se suturó la vena cava inferior con material reabsorbible de polipropileno de 3.0. Para el trombo de nivel III, el hígado fue 
movilizado completamente dividiéndolo entre el ligamento falciforme, triangular y coronario. La circulación colateral fue controlada usando clamps vasculares como en el caso de trombo de nivel II. La arteria femoral y la vena yugular interna se disecaron para llevar a cabo el by pass cardiopulmonar (BCP). Tras ello se introdujo al paciente en una parada circulatoria con hipotermia profunda para poder llevar a cabo la cavotomía y extracción del trombo.

No fue necesaria la realización de esternotomía en estos casos. Para asegurar que todo el trombo había sido extraído, se usó un catéter de Fogarty (tamaño $22 \mathrm{~F}$ y longitud $80 \mathrm{~cm}$ ). Tras ello, la vena renal se seccionó la vena renal y la vena cava inferior fue suturada usando el mismo material que en casos anteriores. Tras el restablecimiento y reparación de la circulación venosa, el by pass fue reanudado y la sangre fue progresivamente calentándose hasta alcanzar el nivel de normotermia, tiempo en el cual el corazón comenzó a latir de nuevo por sî mismo. Las ventanas diafragmáticas fueron construidas para pacientes que necesitaban una desfibrilación interna.

El procedimiento fue similar para pacientes con trombo nivel IV (aurícula derecha), sin embargo se realizó una esternotomía para llevar a cabo el by pass. La aorta y la vena cava inferior se canularon para llevar a cabo el by pass con parada circulatoria en hipotermia profunda. El equipo de cirugía cardiotorácica llevó a cabo la apertura de la aurícula derecha para extraer el trombo. Tras ello se realizó la cavotomía para extraer el resto de trombos. A continuación, se suturó la vena cava inferior, como en casos anteriores, usando material reabsorbible 3.0. Finalmente, se reanudó el by pass y el paciente fue recalentado hasta que se logró la recuperación cardiaca.

Los datos intraoperatorios analizados fueron: acceso, movilización hepática, uso del by pass cardiopulmonar con parada circulatoria e hipotermia profunda, esternotomía y sangrado, transfusión de sangre, morbilidad, mortalidad y tiempo quirúrgico.

Estadio patológico, duración de la estancia hospitalaria y seguimiento (supervivencia global) fueron analizados en el postoperatorio.

Los pacientes fueron seguidos trimestralmente durante el primer año, semestralmente durante el segundo y tercer año y anualmente a partir de ese momento.
Los programas Systat 9.0@ y WinStat@ fueron los usados para el análisis estadístico. Las variables no paramétricas fueron analizadas usando el análisis de la varianza y para las variables categóricas se usó la correlación de Pearson. En todos los test, $\mathrm{P}$ inferior a 0,05 se consideró como indicador de significación estadística. El método de Kaplan - Meier fue el usado para computar las curvas de supervivencia, con diferencias en supervivencia comparada usando el test log-rank.

\section{RESULTADOS}

En siete años, 15 pacientes con tumor renal asociado a trombo en la vena cava inferior fueron sometidos a nefrectomía radical. La media de seguimiento de estos pacientes era 9,5 meses (0 a 36 meses). La media de la edad era 54,5 $( \pm 16,7)$ años, con un rango entre 16 y 79 años. Doce pacientes (80\%) eran varones y solamente tres eran mujeres (20\%). El tumor era más frecuente en el lado derecho (60\%) que en el izquierdo (40\%).

Las presentaciones clínicas más frecuentes fueron hematuria (35\%), edema de miembros inferiores (35\%), masa palpable (42\%) y dolor (75\%). La triada sintomática no estuvo presente en ningún caso. Doce pacientes fueron estudiados como T3b (80\%) y tres como T3c (20\%).

En la presentación de inicio, cuatro pacientes (26\%) ya tenían enfermedad metastásica. Dos pacientes tenían trombo de nivel I $(13,5 \%)$, seis de nivel II (40\%), cuatro pacientes de nivel III $(26,5 \%)$, y tres de nivel IV (20\%). El bypass cardiopulmonar con hipotermia profunda fue realizado en seis pacientes (40\%); cuatro pacientes con trombo de nivel III fueron intervenidos sin estertonomía (66\%); sin embargo, la estereotomía con bypass cardiopulmonar convencional fue realizada en tres casos con trombo de nivel IV (20\%) para extraer el trombo de la aurícula derecha.

El tiempo medio del by pass cardiopulmonar fue de $54( \pm 15,5)$ minutos, entre 32 y 68 minutos, con una media de pérdida de sangre de $5000 \mathrm{~mL}$, entre 3000 y $8000 \mathrm{~mL}$. En pacientes intervenidos sin la asistencia cardiopulmonar, la media de pérdida de sangre era $3.050 \mathrm{~mL}$, entre 1.000 y $6.000 \mathrm{~mL}$. Comparando la pérdida de sangre por intervanción en estos dos grupos no tiene significación estadística $\mathrm{P}=0,27)$.

Un paciente $(6 \%)$ falleció intraoperatoriamente debido al fracaso ventricular después de la conclu- 
sión by pass cardiopulmonar. El paciente tenía un tumor renal grande, metástasis a distancia y trombo de nivel IV, y fue operado usando by pass cardiopulmonar convencional con hipotermia profunda. Otros dos pacientes (12\%), solamente uno sometido a by pass cardiopulmonar, fallecieron directamente en el postoperatorio, los dos por coagulopatía y sindrome de respuesta inflamatoria (SRS), sin respuesta a medidas clínicas.

Las complicaciones del postoperatorio fueron SRS en $33 \%$ de los pacientes, necrosis tubular aguda en $13 \%$, y trombosis venosa profunda en $6 \%$ y embolia pulmonar en $6 \%$. El estadiaje patológico era pT3b en 10 pacientes (67\%), pT3c en 4 (27\%) y pT4 en uno (6\%). El carcinoma de células convencionales fue el tipo histológico más frecuente, presente en 13 casos (86\%). Solamente había un Tumor de Wilms (7\%) y una neoplasia renal indiferenciada (7\%). Ningún paciente presentaba invasión de la vena cava.

La media de estancia en cuidados intensivos y hospitalización fue $3,6( \pm 2,3) / 16( \pm 5,6)$ en el grupo de by pass cardiopulmonar y $2,1( \pm 0.7) / 11,8( \pm 3,9)$ en el grupo operado sin by pass cardiopulmonar, respectivamente $(\mathrm{p}>0,05)$.

No se detectó asociación entre género $(\mathrm{P}=0,416)$, edad $(\mathrm{P}=0,216)$ o estadiaje de tumor $\left(\mathrm{P}=0,380 ; \mathrm{r}^{2}=\right.$ 0,176 ) con supervivencia global más baja. La extensión del trombo no actuó como factor independiente de mal pronóstico, desde que fueron eliminados completamente $\left(\mathrm{P}=0,174 ; \mathrm{r}^{2}=0,176\right)$.

La supervivencia global general fue $60 \%$ en 36 meses (Fig. 1). Sin embargo, la presencia de ganglios linfáticos o enfermedad metastásica a distancia fue asociado con peores pronósticos, y media de supervivencia de seis meses $\left(\mathrm{P}<0,05 ; \mathrm{r}^{2}=0.620\right)$ (Fig. 2).

Debido a la cantidad menor de pacientes con otros tipos histológicos diferentes a carcinoma de células renales, no fue posible determinar la relación con supervivencia.

El tiempo quirúrgico, hemorragia, esternotomía o by pass cardiopulmonar no fueron asociados estadísticamente con la supervivencia general $(\mathrm{P}>0.05)$.

\section{DISCUSIÓN}

Desde que Berg (1913) llevó a cabo el primer manejo quirúrgico del carcinoma de células renales con extensión tumoral a la vena cava inferior, ha habido grandes avances en la técnica quirúrgica, la anestesia y el desarrollo de unidades de cuidados

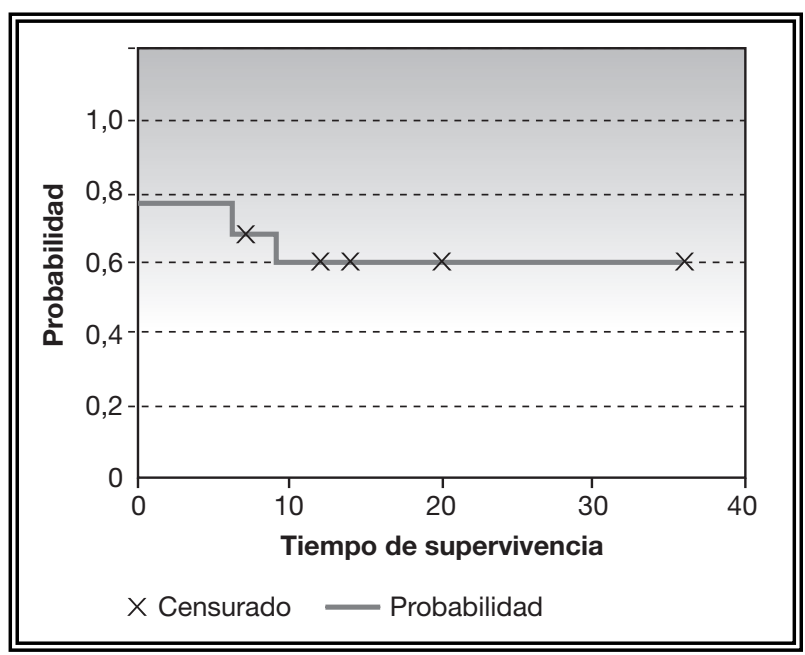

FIGURA 1. Supervivencia global (en meses).

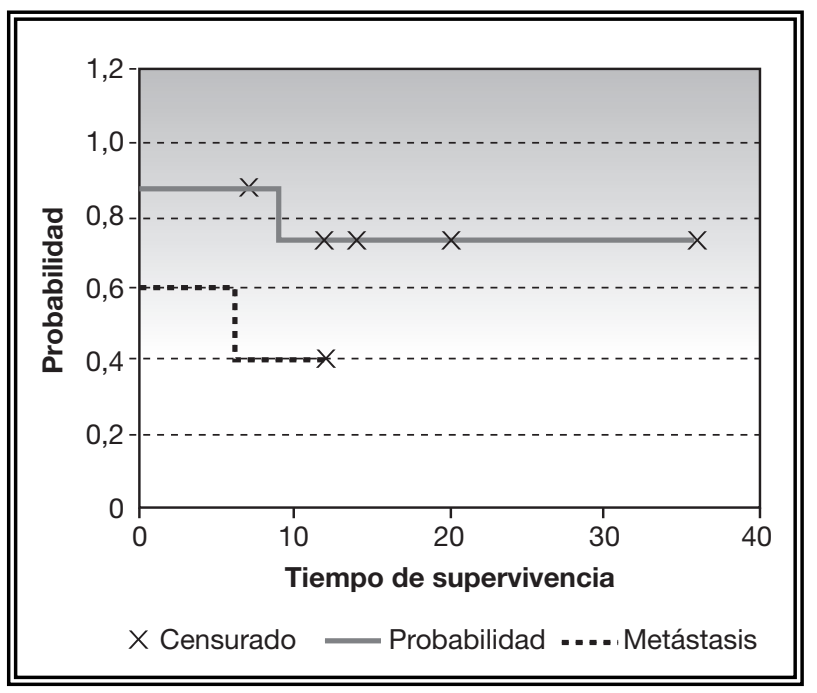

FIGURA 2. Supervivencia de la enfermedad metastásica y no metastásica (en meses).

intensivos, pero el by pass con hipotermia profunda ha sido la piedra angular para enfocar pacientes con tumores renales y trombos en la vena cava inferior $^{10}$. Sin embargo, los refinamientos en la técnica quirúrgica y los avances en la tecnología han sido complementados por la mejoría en las imágenes de resonancia preoperatorio con la finalidad de acceder al tumor y la extensión del trombo ${ }^{9}$. Los estudios de imagen han sido cruciales para definir el manejo quirúrgico.

El bypass ha hecho que las cirugias que no se podían practicar de otra manera, hayan llegado a cirugías de grandes tumores con complejos trombos en la vena cava inferior, reduciendo el sangrado operatorio y en consecuencia las transfusiones de sangre así como el decremento de riesgo de desordenes 
en la coagulación. Por consiguiente, el by pass debe ser usado en las cirugías de tumor renal con trombo extendido hasta la vena cava inferior (clase III o IV), porque las intervenciones de casos complejos serian impracticables sin este aparato. Nuestros datos, de acuerdo con la literatura ${ }^{1-16}$, no mostraron incrementos ni de morbilidad ni de mortalidad con el uso del bypass.

A pesar de que grandes tumores se han reportado en este estudio, la toracofrenolaparotomía no fue necesaria y el acceso quirúrgico usado en nuestros pacientes fue la incisión de chevron bilateral. Cuando fue necesario, la incisión se extendió al apéndice xifoides para un máxima exposición de la vena cava suprahepática, lo que garantiza una gran exposición. Este aspecto particular es debido que nuestro equipo tiene experiencia en cirugía de trasplante hepático y difiere de la literatura actual no teniendo un impacto negativo ni en la morbilidad ni en la mortalidad, evitando así la toracotomía en muchos casos.

El tratamiento quirúrgico radical es la mejor alternativa de tratamiento para este tipo de tumor en el momento actual ${ }^{11,12}$. Pacientes sin enfermedad metastásica tiene un mejor control de la enfermedad con una mayor tasa de supervivencia, con serie que mostraron una tasa de supervivencia a los 5 años de en torno al $68 \%^{1}$, aproximándose a nuestros datos (60\%), lo cual refuerza que estos pacientes deben ser sometidos a procedimiento radical.

El trombo tumoral per se no implica un peor pronóstico, a pesar de que tiene un peor comportamiento biológico ${ }^{1,13-16}$. En estas series, la extensión craneal del trombo no tuvo influencia en la supervivencia, sin embargo, la presencia de ganglios linfáticos y enfermedad metastásica a distancia en el momento del diagnóstico fueron los factores pronósticos principales y los asociados a peor pronóstico de acuerdo con la literatura ${ }^{19-21}$. Algunas series mostraron resultados satisfactorios en pacientes seleccionados con tasas de supervivencia a los 5 años que rondaban entre el 18 y $68 \%{ }^{21}$.

A pesar de que la cirugía abierta es la mejor opción para el tratamiento del cáncer renal con la presencia de trombo, hay estudios mediante video laparoscopia para el tratamiento de trombo de nivel I, con evidencia de buenos resultados y baja morbilidad. Esta vía de acceso está todavía en desarrollo y requiere una futura optimización en un gran volumen de centros, cuyos resultados deben ser publi- cados de forma más consistente a lo largo de muchos centros y su baja morbilidad debería ser confirmada $^{18}$.

Aunque ocurre entre el 4 y $15 \%$ de los pacientes, el trombo tumoral en la vena cava no implica un peor pronóstico para los pacientes ${ }^{3}$. Las variables operatorias analizadas como el tiempo quirúrgico, el sangrado y la necesidad de by pass no alteraron la supervivencia de los pacientes. Sin embargo, algunos estudios asocian la morbilidad y mortalidad de pacientes con variables quirúrgicas en relación con la duración de la cirugía, con peor resultado ${ }^{11}$, lo cual no fue observado en nuestros pacientes.

\section{CONCLUSIONES}

A pesar de su morbilidad y mortalidad, la nefrectomía radical con trombectomía debería ser llevada a cabo en los casos de carcinoma renal con trombo tumoral, porque es el tratamiento más efectivo y puede ofrecer la posibilidad de control local e incremento de supervivencia. Los trombos de nivel IV deberían ser abordados mediante by pass cardiopulmonar. Los pacientes con ganglios linfáticos y enfermedad metastásica a distancia tienen peor pronóstico.

\section{REFERENCIAS}

1. Bastian PJ, Haferkam A, Akbarov I, Albers P, Müller SC. Surgical outcome following radical nephrectomy in cases with inferior vena cava tumor thrombus extension. Journal of Cancer Surgery 2005;31:420-423.

2. O`Donohoe MK, Flanagan F, Fitzpatrick JM, Smith JM. Surgical approach to inferior vena cava extension of renal carcinoma. $\mathrm{Br}$ J Urology 1987;60:492-496.

3. Sweeney P, Wood CG, Pisters LL, Staton JW, et al. Surgical management of renal cell carcinoma associated with complex inferior vena cava thombi. Urologic Oncology 2003;21:327-333.

4. Nesbitt JC, Soltero ER, Dinney CP, et al. Surgical management of renal cell carcinoma with inferior vena cava tumor thrombus. Ann Thoracic Surgery 1997;63:1592-600.

5. Jibiki M, Iwai T, Inoue Y, Sugano N, et al. Surgical strategy for trating renal cell carcinoma with thrombus extending into the inferior vena. Journal of Vascular Surgery 2004; 39(4): 829-35.

6. Swierzewski DJ, Swierzewski MJ, Libertino JA. Radical nephrectomy in patients with renal cell carcinoma with venous, vena caval and atrial extension. Am Journal of Surgery 1994; 168:205-209.

7. Bissada NK, Yakout HH, Babanouri A, Elsalamony T, et al. Longterm experience with management of renal cell carcinoma involving the inferior vena cava. Urology 2003;61(1):89-92.

8. Sosa Re, Mueeke Ec, Vanghan ED, McCarron JP, et al. Renal carcinoma extending into the inferior vena cava: the prognostic significance of the level of vena cava involvement. J Urology 1984; 132:1097-1100.

9. Bussion P, Hakami F, Cordonnier C, Abourachid H. Renal transiotional carcinoma and caval tumor thrombus. Prog Urol 2001; 11(2):288-292. 
10. Berg AA. Malignant hypernephhroma of the kidney, its clinical course and diagnosis, with a description of the author's method of radical operative cure. Surg Gynecol Obstet; 1913; 17:463-471.

11. Zisman A, Wieder JA, Pantuck AJ, Chao DH, et al. Renal cell carcinoma with tumor thrombus extension: biology, role of nephrectomy and response to immunotherapy. J Urology 2003; 169:909-916.

12. Staehler G, Brokovic D. The role of radical surgery for renal cell carcinoma with extension into the vena cava. J Urology 2000; 163:1671-1675.

13. Hatcher PA, Anderson EE, Paulson DF, Carson CC, et al. Surgical management and prognosis of renal cell carcinoma invading the vena cava. J Urology 1991;145:20-24.

14. Mounzadeh A, Libertino JA. Prognostic significance of tumor thrombus level in patients with renal cell carcinoma and venous tumor thrombus extension. Is all T3b the same? $\mathrm{J}$ Urology 2004;171:598-601.

15. Glazer AA, Novick AC. Long-term follow-up after surgical treatment for renal cell carcinoma extending into the right atrium. J Urology 1996;155:448-50.

16. Kim HL, Zisman A, Han KR, Fiflin RA, et al.Prognostic significance of venous thrombus in renal cell carcinoma. Are renal vein and inferior vena cava involvement different? J Urology 2004; 171:588-591.
17. Langenburg SE, Blackbourne LH, Sperling JW, Buchanan SA, et al. Management of renal tumors involving inferior vena cava. Journal of Vascular Surgery 1994;20:385-388.

18. Steinnerd LE; Vardi IY, Bhayani SB. Laparoscopic radical nephrectomy for renal carcinoma with known level I renal vein tumor thrombus. Urology 2007;69(4):662-665.

19. Bensalah K; Guille F; Vincendeau S; Rioux-Leclercq N, et al.Clinical and histological porgnostic factors of renal câncer with caval thrombus. Prog Urol 2004;14(2):160-166.

20. Martinez-Salamanca JI, Herranz F, Verdu F, Pendemonte G, et al. Surgical tratment of renal adenocarcinoma with venous thrombus. Arch Esp Urol 2005; 58(4):295-304.

21. Klatte T, Pantuck AJ, Riggs SB, Kleid MD, et al. Prognostic factors for renal cell carcinoma with tumor thrombus extension. J Urol 2007;178(4 Pt 1):1189-1195.

Correspondencia autor: Dr. Leonardo Oliveira Reis

Sección de Urología Oncológica. Servicio de Urología. Universidad de Campinas. Unicamp-Campinas, Brasil

Tel.:/Fax: + 5519 3521-7481

E-mail autor: reisleo@unicamp.br

Información artículo: Original - Cáncer renal

Trabajo recibido: diciembre 2008

Trabajo aceptado: enero 2008 